\title{
Kernos
}

Revue internationale et pluridisciplinaire de religion grecque antique

$3 \mid 1990$

Varia

\section{"Je suis devin » (Phèdre, 242c). Remarques sur la philosophie selon Platon}

\section{Richard Bodéüs}

\section{OpenEdition \\ Journals}

\section{Édition électronique}

URL : http://journals.openedition.org/kernos/969

DOI : $10.4000 /$ kernos.969

ISSN : 2034-7871

\section{Éditeur}

Centre international d'étude de la religion grecque antique

\section{Édition imprimée}

Date de publication : 1 janvier 1990

ISSN : 0776-3824

\section{Référence électronique}

Richard Bodéüs, " « Je suis devin » (Phèdre, 242c). Remarques sur la philosophie selon Platon », Kernos [En ligne], 3 | 1990, mis en ligne le 19 avril 2011, consulté le 02 mai 2019. URL : http:// journals.openedition.org/kernos/969; DOI : 10.4000/kernos.969 


\section{«JE SUIS DEVIN" (Phèdre, 242c). REMARQUES SUR LA PHILOSOPHIE SELON PLATON}

Au regard du rationalisme contemporain, le triomphe de la philosophie, en Grèce, marque la ruine de la divination. Platon, parmi les philosophes grecs, n'autorise pas, cependant, un jugement aussi net. Et le platonisme, au terme de l'Antiquité, passe encore pour confirmer la foi aux oracles, au lieu de l'infirmer. L'étude de ce phénomène, lié à l'interprétation des textes de Platon, exigerait un lourd dossier. Ce n'est pas l'objet des brèves remarques qui vont suivre. Celles-ci se bornent à prendre en compte les principales données sur la divination qui, chez Platon lui-même, éclairent le statut de la philosophie et de son discours. Encore, vu l'espace alloué, a-t-il fallu faire l'économie des discussions suscitées par l'énorme littérature savante consacrée à chacun des points abordés. C'est au prix d'un tel sacrifice - hélas! - que l'on peut aujourd'hui viser, dans des limites aussi étroites, à un exposé de synthèse qui profite aux recherches interdisciplinaires.

Les historiens de l'Antiquité distinguent habituellement deux sortes de divination : l'une «inductive», l'autre "inspirée». En un sens, elles s'opposent diamétralement. Tandis que la première (illustrée par l'«oiônistique» et l'«hépatoscopie», notamment) prétend s'appuyer sur une véritable science (celle des signes), la seconde, en revanche (caractérisée par l'«enthousiasme»), table sur une révélation où entre si peu de science qu'elle paraît l'apanage de gens simples en délire. Un abîme sépare les deux formes de divination ainsi distinguées. Si l'oracle des inspirés se donne à croire sans justification, celui des autres devins s'autorise, en effet, de prémisses à partir desquelles, par mode d'inférences, les signes sont décodés. Avec cette prétention, la divination inductive s'exposait à une critique ruineuse de la part de la pensée rationnelle. Une science ne vaut, en effet, que ce que valent ses prémisses. Que valaient celles des devins ? Peu de chose. Les progrès de la pensée scientifique mirent en doute qu'ils eussent la science des signes. Paradoxalement, malgré son allure irrationnelle, la divination des inspirés résistait mieux aux progrès de la même pensée scientifique. La parole crue sans raison se vérifiait-elle à l'occasion, force était d'admettre que l'on pût dire vrai sans véritable savoir. Le fait paraît s'être imposé au regard de la philosophie classique. Platon ne feint pas en plaçant la folie divinatoire au-dessus des formes ration- 
nelles de divination (Phèdre, $244 \mathrm{a}-\mathrm{b})$ : il y a place pour des opinions qui soient vraies.

La philosophie, à la fin de l'Antiquité, prit cet hommage très au sérieux. C'est au point que les néo-platoniciens voulurent faire coïncider vérité oraculaire et vérité philosophique : aucune différence, pour eux, entre ce que la divinité prend l'initiative de révéler aux inspirés et ce que le philosophe, lui aussi inspiré à sa façon, saisit de la réalité intelligible ou de son principe trans-intelligible. La philosophie confirme les oracles et réciproquement.

Platon distinguait pourtant ce que ses émules tendaient à fondre. Et si la folie du philosophe (qu'il appelle "érotique») ressemble à la folie du devin inspiré, ce n'est pas à dire, selon lui, que l'un et l'autre puisent directement à la même source, ni produisent la vérité relativement aux mêmes choses. Il s'en faut de beaucoup. Et, comme on va le voir, le phénomène de la divination n'est, pour Platon, qu'un modèle permettant de (se) représenter la philosophie telle qu'il la conçoit. Le privilège de la divination inspirée est ainsi d'entrer dans l'œuvre de Platon à titre de paradigme pour instruire analogiquement sur ce que doit être la philosophie.

Du phénomène de la divination, Platon rend compte physiologiquement (dans le Timée, 71a-72c) par la présence, en l'homme, d'un organe approprié, le foie, où peuvent se lire, en quelque sorte, des signes à interpréter. Le philosophe semble de la sorte viser, non la divination inspirée, mais la science divinatoire (par signes). Il n'en est rien. C'est bien de l'inspiration qu'il s'agit et des paroles proférées par enthousiasme. Platon explique, en effet, que celles-ci traduisent les visions qu'une âme, dont l'esprit est troublé par le sommeil, la maladie ou le délire, perçoit intérieurement et que laisse apparaître la surface du foie. Quant au sens de ces visions et des discours qui les expriment, il n'appartient pas, selon Platon, à l'inspiré de le découvrir. C'est l'affaire de l'esprit qui raisonne. Le philosophe, en d'autres termes, soustrait à la compétence du devin, la science des signes. Il n'est pas au pouvoir du devin, dépourvu d'intelligence, d'interpréter des signes. Mais, en vertu du même défaut, qui s'avère en l'occurrence aussi un avantage, il est en son pouvoir de voir des signes par ailleurs inaccessibles. Telle est la mantique devant laquelle le philosophe s'incline, comme devant une source de vérité, invoquant les bienfaits, pour l'âme irrationnelle, de la physiologie hépatique! A cet égard, le privilège de la vraie mantique est de disposer en somme de bons miroirs de la vérité. Vision de reflets $\left(\varepsilon \varepsilon^{\prime} \delta \omega \lambda \alpha\right.$ ), la divination peut nous instruire, si elle 
exprime des reflets ou des images peu déformées sur l'écran que constitue un foie lisse et brillant. Cela suppose, en amont, une source d'images, qui, pour Platon, n'est autre que le flux des pensées issues de l'intellect. Les images ( $\varphi \alpha v \varepsilon ́ v \tau \alpha / \varphi \alpha \nu \tau \alpha \dot{\sigma} \sigma \mu \alpha \tau \alpha)$ sont le reflet de ces pensées $\left(\delta\left\llcorner\alpha \vee \eta^{\prime} \mu \alpha \tau \alpha\right)\right.$. Cela suppose aussi, en aval, une autre intelligence : celle du prophète, capable, dit Platon, de déchiffrer le sens de la vision divinatoire exprimée, c'est-à-dire de comprendre ce dont la vision exprimée est le signe seulement ( $\sigma \eta \mu \alpha i ́ v \varepsilon \imath$ ). Mais le privilège des inspirés - la Pythie de Delphes, les prêtresses de Dodone, la Sybille, ... que Platon mentionne ailleurs (Phèdre, 244a-b) comme illustrations du délire divinatoire - est strictement de voir et de dire des signes, où se reflète une vérité qui peut être saisie par interprétation.

Malgré les apparences peut-être, nous sommes ici en présence d'un phénomène complexe. Quatre traits sont à épingler. 1) Le devin est un visionnaire, ayant accès à ce que le commun ne voit pas. 2) Dans cet état de visionnaire, le devin se trouve hors de soi, hors de son bon sens. 3) Son discours, à l'instar de la vision qu'il exprime, n'est pas, comme tel, directement intelligible. 4) Néanmoins, il a du sens, il est révélateur de la vérité, pour qui sait l'interpréter. Ces quatre traits, sous lesquels apparaît le phénomène de la divination décrit par Platon, n'ont pas seulement la vertu de préciser les contours de la mantique véritable aux yeux du philosophe. Parce que le phénomène en question constitue une forme d'appréhension et d'expression de la vérité, ils donnent aussi à se représenter analogiquement quatre dimensions du phénomène qu'est la philosophie, forme par excellence de l'appréhension et de l'expression du vrai.

La dimension «maniaque» ou délirante de la philosophie (second trait de la divination) est la plus évidente pour le lecteur de Platon. Elle n'est peut-être pas, cependant, la plus significative.

On sait que Platon rapproche explicitement la divination d'autres formes de délire : le délire poétique et le délire érotique, notamment (Phèdre, 244a-245c; 265b). Ce dernier est le fait de quiconque aperçoit l'imitation de la Beauté sur un visage humain qui la reflète (251a). Le "flux de la Beauté" idéale (251b), comme le flux des pensées qui viennent frapper le foie, renvoie à l'éromène le courant. Dans son éraste, dit Platon, l'éromène se voit "comme dans un miroir» et lui retourne une «image d'amour» (255c) - Reflet de reflet ! Le délire poétique, notons-le au passage, comporte le même phénomène de sympathie communicative, que Platon décrit comme une chaîne d'anneaux magnétiques (Ion, 533c-536d) : du poète inspiré au rhapsode et 
du rhapsode à ceux qui l'écoutent, ... se transmet l'enthousiasme que suscitent les images.

Au cœur de tout cela, sans doute, la même présence d'images entrevues, signes d'une réalité intelligible qui met hors de soi. De ces sortes de "manies", dont la folie divinatoire est l'exemple le plus spectaculaire, Platon n'hésite donc pas à rapprocher la philosophie, mais dans son état naturel. Le privilège d'une nature philosophique, en effet, est aussi de voir des signes et de céder à leur envoûtement comme aux charmes de l'amour. Mais, à la différence du devin, des autres insensés et même de l'amoureux ordinaire, le philosophe ne subit pas totalement l'ascendant d'un signifiant inintelligible ou d'un reflet sensible. La séduction qu'exerce sur lui ce qu'il voit, ne l'entraîne pas, comme

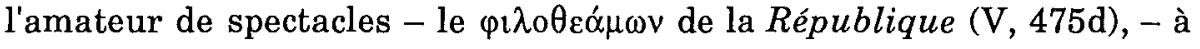
donner au signe les faveurs que mérite cela seulement dont il est signe. Ce qui attire consciemment ce $\varphi \imath \lambda o \mu \alpha \theta \eta \dot{s}$ qu'est le philosophe, c'est l'intelligible signifié, dont le sensible éveille en lui le souvenir (Phèdre, 251a).

À cette différence, qui sépare le devin du philosophe au naturel, s'ajoute une seconde différence qui, cette fois, sépare le devin du philosophe parvenu à (reconquérir) l'intelligence du réel séparé. L'infirmité du devin qui voit les signes, mais ne peut dire ce qu'ils signifient sans le secours d'un prophète, s'oppose à l'autorité du philosophe qui, tel le prophète pour les paroles du voyant, est en mesure d'interpréter ce qu'il voit à la lumière de ce qu'il sait. La supériorité du philosophe sur le devin - qui est celle de l'intelligence sur la sensation - se trouve compensée par une sorte d'infériorité. Car le philosophe ne voit pas les signes que voit le devin dans son miroir et qui sont les reflets d'une intelligence "d'un bien ou d'un mal futur, passé ou présent» (Timée, 72a). D'autre part, même si Platon ne le dit pas, tout porte à croire qu'à son estime, le philosophe n'a non plus aucun avantage sur le prophète. Car l'intelligence que celui-ci déploie en déchiffrant le sens rationnel des signes entrevus par le devin est une intelligence discursive du devenir, bref une intelligence toute pratique, à laquelle le philosophe ne donne aucun soin particulier. Celui-ci ferme, en effet, les yeux au cours du monde et se hisse à la contemplation de l'immuable.

Au total, le philosophe que met hors de soi l'image de la Beauté entrevue, se soustrait à l'emprise des images sensibles, dont le flux ne lui importe pas. Il déchiffre l'éternel dans le mouvant et non, comme le prophète, la certitude dans le mouvement dont on lui représente approximativement le cours. Il ne devine ni l'origine ni l'issue d'un 
devenir sensible. Il appréhende seulement son fondement intelligible séparé. Par où l'on voit le monde qui sépare, selon Platon, l'inspiration divinatoire et l'inspiration philosophique, que ses émules prétendaient fondre dans l'unité, au mépris de la différence entre la vérité du discours qui dit adéquatement le devenir et celle du discours qui prononce adéquatement sur son principe séparé.

Mais le philosophe, qui, par ce trait, s'éloigne du devin, s'en rapproche par un autre : le premier que nous avons signalé en commençant. La contemplation philosophique, en effet, n'est pas sans analogie avec la vision divinatoire. Le philosophe, pour Platon, est entraîné à voir $(\theta \varepsilon \omega \rho \varepsilon \hat{\imath} v)$ comme le devin. De même que le devin voit sans intelligence des signifiants, le philosophe voit, par l'intelligence, des signifiés. C'est un visionnaire dans l'ordre de l'intelligible. Il est inutile, pensons-nous, d'insister sur cette ressemblance, qui conduit

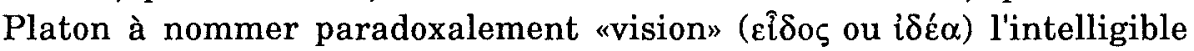
lui-même.

Cet usage paradoxal est néanmoins indicatif. En nommant l'objet purement intelligible à l'aide d'un mot qui, contradictoirement, semble renvoyer au sensible vu, le discours philosophique n'échappe pas à une sorte d'irrationalité qui l'apparente au discours divinatoire pour celui qui l'entend. Le philosophe, en effet, ne se contente pas d'être un voyant: il parle, comme le devin. Et, sous ce rapport, la philosophie semble présenter une physionomie où se reconnaissent les troisième et quatrième traits de la mantique. Incompréhensible en lui-même, parce qu'il fait signe seulement vers l'intelligible, le discours philosophique n'en demeure pas moins significatif pour l'intelligence d'autrui à certaines conditions.

On touche ici à un point capital du platonisme, pour qui l'intelligibilité n'est point une caractéristique du discours, lequel se borne à la démarquer en la trahissant dans une image. Le philosophe qui parle ne procède pas comme le devin. Mais il aboutit à un résultat qui n'est pas très différent. Le devin dit les images qu'il voit. Le philosophe, lui, dit ce qu'il voit dans les images. Il procède, en fait, à la façon du poète inspiré, visionnaire lui aussi.

Un mot là-dessus est indispensable. Le bon poète ne fait point fondamentalement œuvre d'intelligence (Phèdre, 245a). Il ne sait pas ce qu'il dit lorsqu'il vaticine (Ion, 534b-d). Aveugle à l'intelligible, auquel le ferme son délire, il est aussi aveugle au sensible qu'il a sous les yeux. Et, sous ce rapport, le poète s'apparente au devin : ce qu'il voit, semble-t- 
il, ce sont des images. Son discours n'est pourtant pas exactement celui du devin stricto sensu, qui, fidèlement et sans intelligence, reproduit des images entrevues ou des paroles entendues. Le poète, nous dit Platon, est "l'interprète» des dieux (Ion, 534e), mais à la qualité de visionnaire, qui fait le pur devin, il allie la technique de l'art, qui fait aussi de lui un imitateur. Or tout porte à croire que l'imitation poétique tend, pour Platon, à dégrader le modèle imité. Le poète qui fabrique une imitation d'image trahit ainsi l'intelligibilité que reflète l'image paradigmatique entrevue. Que voit, en effet, sans intelligence le poète hors de soi ? Par définition, ce n'est pas le modèle intelligible du vivant, à l'image duquel est fabriqué le monde. Ce n'est pas non plus les vivants sensibles qu'il pourrait avoir sous les yeux et qui sont l'imitation la plus dégradée qui soit du vivant idéal. Qu'est-ce alors? Le Timée nous souffle, semble-t-il, une réponse précise que l'on peut hasarder. C'est l'imitation de l'intelligible séparé, que sont les premiers vivants parfaits fabriqués par le démiurge : les dieux (visibles, dans le ciel, ou invisibles, mais qui se manifestent à leur gré), ainsi que l'imitation de cette première imitation que sont les hommes parfaits, fabriqués par le démiurge et les dieux eux-mêmes. Le privilège du bon poète, c'est de voir (ou d'imaginer) les dieux tels qu'ils sont et les hommes divins, tels qu'en eux la Beauté se reflète. De cette image saisie sans intelligence, c'est-à-dire, sans l'intellection de ce qu'est un dieu ou un homme en soi-même, le poète tend à donner, dans son discours, une imitation qui la dénature : les dieux, chez lui, deviennent humains et les hommes perdent leur beauté divine. C'est pourquoi Platon, dans la République (II, 379a), par exemple, croit utile d'indiquer aux poètes, les

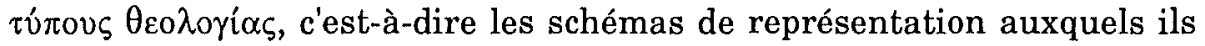
doivent se conformer pour chanter les dieux. Un passage des Lois (IV, $719 \mathrm{c})$ résume le jugement du philosophe : «Chaque fois que le poète se trouve assis sur le trépied des Muses, il est hors de sens et ressemble à une fontaine qui donne libre cours au flot des eaux qui le submerge. Et, comme son art réside dans une imitation, il se trouve entraîné souvent à se contredire, créant des caractères d'humeurs contradictoires et ne sachant laquelle de ces expressions contradictoires est vraie.»

Nous avons dit plus haut que le philosophe, lorsqu'il parle, procède à la manière du poète. Ce n'est point seulement quand il use d'un langage poétique, fabrique des histoires et imite le devenir dans des mythes. Au contraire. Car l'on comprend immédiatement qu'ayant l'avantage d'un accès à l'intelligible, le philosophe est en mesure, lui, de dénoncer les contradictions du poète. Il est en mesure aussi de réformer les images du poète à la lumière de l'intelligible et de créer ainsi son propre 
discours poétique, d'où peut se trouver éliminée la contradiction interne. Fabriquée à partir de la réalité intelligée et non plus sur le modèle de son imitation entrevue par la sensibilité exceptionnelle du poète, l'imagerie du philosophe n'en demeure pas moins une imitation, où la vérité n'est donnée que partiellement, mêlée de choses qui s'en écartent, ainsi que le confesse Platon lui-même (Phèdre, 256a-c : à propos de l'«hymne mythique» sur l'amour). La réalité ne s'y trouve pas dite, mais suggérée ou plutôt signifiée, entendez : évoquée dans un signe qui, en lui-même et quoi qu'on fasse, trahit la réalité autant qu'il ne l'évoque. Image de l'âme, l'attelage ailé dit autre chose que l'âme. Mais, pour Platon, cela est vrai, non seulement du langage poétique, mais de tout langage, qui imite les choses signifiées d'une manière qu'on ne peut jamais dire sûrement adéquate. Comme l'explique à suffisance le Cratyle (423b et sq.), le langage, par lui-même, n'indique pas sûrement ce que sont les choses en vérité. Ce n'est pas que le philosophe, usant du langage, soit incapable de s'exprimer en vérité, c'est-à-dire d'une manière conforme à la réalité intelligible qu'il connaît. Il peut substituer au réel les mots qui lui correspondent vraiment ou qui l'imitent bien. Mais aucun discours vrai n'est, pour celui qui l'écoute, un reflet certain de la réalité, parce que l'intelligibilité n'est pas une propriété du discours lui-même, mais de la chose qu'il reflète. Et rien dans le discours vrai n'indique à celui qui l'écoute que celui qui parle exprime autre chose qu'une opinion faillible, comme le législateur des noms primitifs (Cratyle, 436c). Bref, chacun éprouve, à reconnaître la vérité au-delà du langage qui la signifie éventuellement, la même difficulté que le philosophe à la faire reconnaître par delà son propre langage. Or le philosophe veut que cela puisse être reconnu!

Et c'est cette difficulté qui, chez Platon, invite le discours philosophique à prendre volontairement un trait du discours oraculaire. Loin, en effet, de produire un discours d'où serait exclue la contradiction qu'il reproche à l'ignorance du poète, le philosophe, au contraire, construit son discours sur une contradiction, élaborée en pleine connaissance de cause. Le philosophe ne tait pas ce qu'il sait non conforme à la réalité, mais l'oppose dialectiquement au discours vrai. Pour que paraisse la réalité intelligible que celui-ci reflète, le philosophe n'a d'autre ressource que de le faire contredire par un autre discours. Le Phèdre offre du procédé un exemple admirable. Il faut, à l'accréditation de la thèse, une anti-thèse. 
Sommé par son compagnon de parler ( $\dot{\eta} \tau \varepsilon \dot{c} 0 v: 236 c$ ), comme un devin que l'on presse de répondre à une question, Socrate s'exécute et produit un double discours contradictoire : le premier placé sous l'invocation des Muses, le second donné pour la palinodie de Stésichore. L'ambiguitté de cette réponse, qui peut faire songer aux oracles de Loxias, est évidemment levée formellement par des indices extérieurs aux discours. Prévenu par le signe démonique, Socrate avertit que la

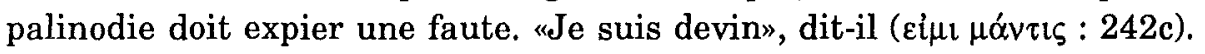
Le second essai oratoire doit donc être plus vrai que le premier. Mais comment Phèdre, lui, peut-il, en dehors de cet avertissement, reconnaître la vérité du discours palinodique expiatoire sans être déjà philosophe et en possession de la vérité ? Il ne le peut pas et le lecteur du dialogue ne le peut pas non plus. Sur la foi d'indications extérieures, chacun peut croire que ce discours est vrai. Mais en lui-même, ce discours ne contient que les signes de la vérité. Or quels sont ces signes ? À quoi paraissent-ils ? Ils ne paraissent, en définitive, que par l'effet de l'opposition entre deux discours contradictoires, laquelle permet de faire ressortir la supériorité du second sur le premier. Ce qui ressort ainsi par contraste, c'est, en réalité, l'excessive beauté

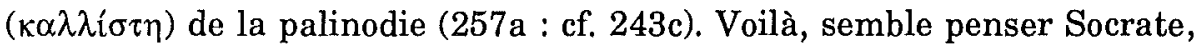
le signe par excellence de la vérité : la Beauté se reflète dans le discours et témoigne en faveur de ce discours.

Le philosophe n'ignore pas de quel côté se trouve la vérité et il prend soin d'en avertir son auditeur. Encore faut-il que celui-ci puisse éprouver la vérité que, par lui-même, le discours n'enseigne pas. Encore faut-il, ajoutera-t-on, que le lecteur du philosophe, comme le prophète du devin, puisse décoder les signes. Mais qu'est-ce ici que cette critique du sens? Il ne s'agit pas de trancher entre deux discours contradictoires. Il s'agit de saisir, par delà la contradiction, la source de toute vérité, dont le discours est partiellement le reflet.

Dans le jeu qu'est pour lui l'écriture, Platon ne veut point se faire entendre, mais, tel le devin, faire entendre une voix dont il n'est luimême que le truchement. Aucun des personnages derrière lesquels le philosophe s'efface dans son œuvre ne parle en son propre nom. Leurs paroles ne sont pas plus de leur auteur que les oracles de Delphes n'étaient de la Pythie.

Université de Montréal

Richard BODÉÜS

Département de philosophie

C.P. 6128 , succ. A

MONTRÉAL (QUÉBEC) H3C 3J7

Canada 